\title{
Melanoma vaccines and viral-based immunotherapies: a special focus issue from Melanoma Management
}
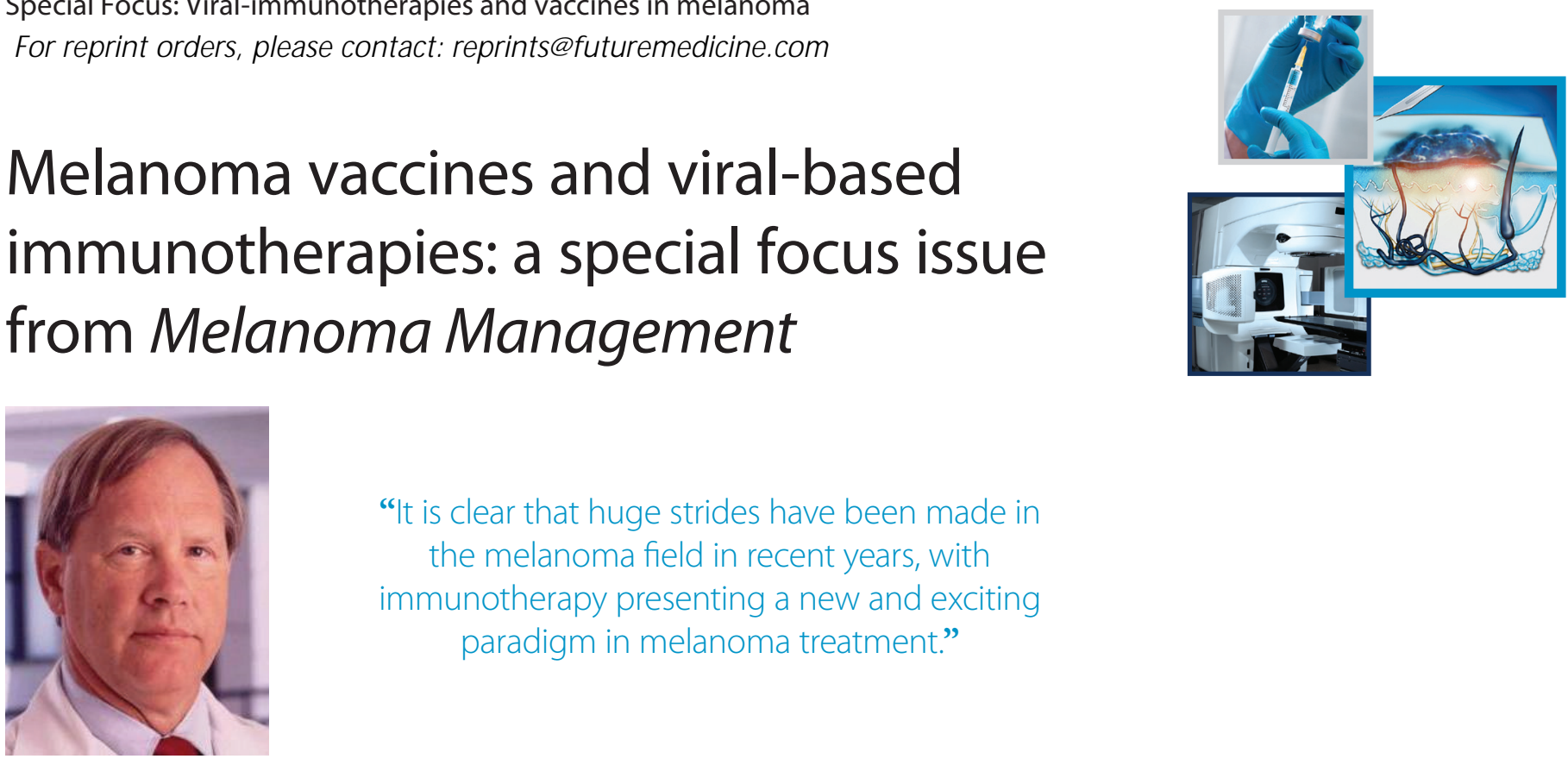

\author{
"It is clear that huge strides have been made in \\ the melanoma field in recent years, with \\ immunotherapy presenting a new and exciting \\ paradigm in melanoma treatment.”
}

\section{Robert O Dillman ${ }^{*, 1} \&$ Sebastian Dennis-Beron ${ }^{2}$}

First draft submitted: 6 September 2016; Accepted for publication: 8 September 2016; Published online: 30 November 2016

Metastatic melanoma historically was best managed through surgical resection whenever possible, and various relatively ineffective systemic therapies. However, in recent years, immunotherapy has emerged as an effective approach to melanoma treatment, with monoclonal antibodies, such as anti-CTLA-4 and anti-PD-1, receiving US FDA approval for treatment of late-stage melanoma. Moreover, we are now recognizing the advancements being made in potential cancer vaccines using tumor-associated antigens and intralesional viral therapies, which are currently providing exciting results.

This special focus issue of Melanoma Management highlights the latest advancements in melanoma immunotherapy with respect to cancer vaccines and viral-based therapies, with a range of articles discussing the developments in various vaccine candidates for melanoma as well as expert opinions and experiences with such vaccines both in clinical trials and in standard practice.

Dendritic cell (DC) vaccines are a promising form of cancer vaccine that load the antigen presenting immune cells with tumor-associated antigen ex vivo. Dillman and colleagues provide a detailed review of DC vaccines, discussing the results seen in previous clinical investigations, current questions being posed in the field regarding the vaccine and potential future directions for the therapy [1].

Bol et al. builds on DC vaccines by disseminating results from various $D C$ vaccine clinical trials to analyze the state of progression with the cancer vaccine at this moment in time. Bol and colleagues discuss various aspects of the treatment in terms of practical applications in the clinic, building on what has been reported by clinical trials and suggesting possibilities for DC vaccine development in concert with other immunotherapies [2].

Clinical trial results for cancer vaccines, not limited to $\mathrm{DC}$ vaccines, are put into perspective in an editorial by Riker et al., where the authors critique the reports from various clinical trials and translate those results to a clinical level, including previous experiences with using the treatments in practice [3].

\section{KEYWORDS}

- dendritic cell vaccine

- immunotherapy

- melanoma • neoantigen

- vaccine 
“...we employed two novel systems that better mimic the patient situation than conventional cell lines: ex vivo cultured metastatic melanoma slices and 3D melanoma cultures, dubbed melanoids."
Storkus and colleagues, further appraise cancer techniques discussing both the promises and limitations involved in the treatment [4]. Storkus et al. further detail vaccine targets in melanoma, targeting the treatment toward antiangiogenic tumor vascularization. The editorial piece concludes by looking at the potential use of the kinase inhibitor Dasatinib as a treatment for advanced melanoma in combination with vaccines.

Focusing on viral therapies, Zager et al., provide a review on talimogene laherparepvec (T-VEC), an oncolytic immunotherapy currently used as an intralesional treatment for advanced melanoma. Zager and colleagues provide an in-depth overview of the therapy, providing detailed information regarding T-VEC clinical trials and current practices. Finally, the review takes a look at potential applications for T-VEC in the future, with the authors suggesting that it may be used in combination with check-point inhibitors [5].

A special report, by Johnson and Wang, covers a wide range of intralesional therapies, such as PV-10 (Rose Bengal), IL-12 plasmid electroporation and Coxsackievirus A21 as well as T-VEC [6]. The authors evaluate the various therapies, reporting clinical trial results and their use in practice. The report also critically assesses future directions for these therapies, and as with cancer vaccines, their potential in combination with monoclonal antibody inhibitors.
It is clear that huge strides have been made in the melanoma field in recent years, with immunotherapy presenting a new and exciting paradigm in melanoma treatment. This special issue puts the latest developments in the field into context and provides a forwardlooking perspective on the potential of this relatively underexplored therapeutic approach. I very much hope that you enjoy the issue and look forward to hearing your thoughts on this important topic.

Despite these advances, we are still pushing forward toward the development of cancer vaccines and intralesional viral therapies, which either as monotherapy or in combination with other immunotherapeutics - could revolutionize the way melanoma is treated.

We look forward to hearing your thoughts on this important topic, and welcome future submissions to Melanoma Management addressing this issue.

Financial \& competing interests disclosure The authors have no relevant affiliations or financial involvement with any organization or entity with a financial interest in or financial conflict with the subject matter or materials discussed in the manuscript. This includes employment, consultancies, honoraria, stock ownership or options, expert testimony, grants or patents received or pending, or royalties.

No writing assistance was utilized in the production of this manuscript.

\section{References}

1

AN. Dendritic cell vaccines for melanoma: past, present, and future. Melanoma Manag. 3(4), $\mathrm{xxx}-\mathrm{xxx}(2016)$.

2 Halilovic A, Bol KF. The use of dendritic cell vaccinations in melanoma: where are we now? Melanoma Manag. 3(4), xxx-xxx (2016).
3 Riker AI, Bisgaard E. Current clinical trials for melanoma vaccines: where do we stand? Melanoma Manag. 3(4), xxx-xxx (2016).

4 Tarhini A, Tawbi H, Storkus WJ. Vaccine therapy + dasatinib for the treatment of patients with stage IIIB-IV melanoma. Melanoma Manag. 3(4), xxx-xxx (2016).
5 O’Donoghue C, Doepker MP, Zager JS. Talimogene laherparepvec (T-VEC): overview, combination therapy and current practices. Melanoma Manag. 3(4), xxx-xxx (2016).

6 Wang DY, Johnson DB. Advances in the development of intralesional therapies for melanoma. Melanoma Manag. 3(4), xxx-xxx (2016). 